\title{
Effect of Bile Duct Ligation-induced Liver Dysfunction on Meth- amphetamine Pharmacokinetics and Locomotor Activity in Rats
}

\begin{abstract}
Michael D. Hambuchen ${ }^{1,2}$, Michael D. Berquist ${ }^{2}$, Christy M. Simecka ${ }^{3}$, Mitchell R. McGill ${ }^{4}$, Melinda G. Gunnell ${ }^{2}$, Howard P. Hendrickson ${ }^{5,6}$, and S. Michael Owens ${ }^{2}$

${ }^{1}$ Department of Pharmaceutical Science and Research, Marshall University School of Pharmacy, Huntington, WV, USA, ${ }^{2}$ Department of Pharmacology and Toxicology, College of Medicine, University of Arkansas for Medical Sciences, Little Rock, AR, USA, ${ }^{3}$ Division of Laboratory Animal Medicine, University of Arkansas for Medical Sciences, Little Rock, AR, USA, ${ }^{4}$ Department of Environmental and Occupational Health, Fay W. Boozman College of Public Health, University of Arkansas for Medical Sciences, Little Rock, AR, USA, ${ }^{5}$ Department of Pharmaceutical, Social, and Administrative Sciences, McWhorter School of Pharmacy, Samford University, Birmingham, AL, USA, ${ }^{6}$ Department of Pharmaceutical Sciences, College of Pharmacy, University of Arkansas for Medical Sciences, Little Rock, AR, USA.
\end{abstract}

Received, April 21, 2019; Revised, July 6, 2019; Accepted, July 9, 2019; Published, July 10, 2019

\begin{abstract}
Purpose: Methamphetamine (METH) abuse is associated with hepatic dysfunction related comorbidities such as HIV, hepatitis $\mathrm{C}$, and polysubstance abuse with acetaminophen-containing opioid formulations. We aimed to develop a bile duct ligation (BDL)-induced hepatic dysfunction model for studying both METH and experimental treatments for METH abuse in this comorbidity. Methods: Sham or BDL surgery was performed in male Wistar rats on day 0 . Liver function was measured throughout the study. On days 7 and 19, serum pharmacokinetics studies were performed with $1 \mathrm{mg} / \mathrm{kg}$ subcutaneous (sc) METH. On day 21, this dose was repeated to determine $2 \mathrm{~h}$ post-METH brain concentrations. METH-induced open field behaviors were measured every other day (days 12 - 16) with ascending sc doses $(0.3-3 \mathrm{mg} / \mathrm{kg})$. Results: BDL transiently increased alanine aminotransferase levels and altered liver structure, which resulted in significantly greater METH serum and brain exposure. In the BDL compared to sham group, there was a longer duration of METH-induced locomotor activity (after 1 and $3 \mathrm{mg} / \mathrm{kg}$ ) and stereotypy (after $3 \mathrm{mg} / \mathrm{kg}$ ). Conclusions: In rats, liver dysfunction reduced METH clearance, increased brain METH concentrations, and enhanced METH effects on locomotor activity in a dose dependent manner. In addition, this model could be further developed to simulate the associated hepatic dysfunction of key METH abuse comorbidities for preclinical testing of novel pharmacotherapies for effectiveness and/or toxicity in vulnerable populations.
\end{abstract}

\section{INTRODUCTION}

While government action substantially reduced both the number of clandestine methamphetamine (METH) labs and METH use in the United States, METH-related deaths and DEA seizures are once again on the rise (1). This rise is a concern as METH use disorders are extremely difficult to treat; even keeping individuals enrolled in highly organized clinical treatment trials is incredibly challenging (2). In addition, there are currently no FDA-approved medications for this chronic illness. Small molecule and biologic therapies are in development for METH use disorder $(3,4)$ and are being tested in human trials $(5,6)$. Although preclinical studies have found that small molecule and biologic therapies are effective in healthy animal models, METH use is associated with a variety of comorbidities, such as HIV infection (7), hepatitis C infection (8), and polysubstance use with acetaminophen containing prescription opioid formulations (9). A common characteristic of these comorbid conditions is hepatic dysfunction. While acetaminophen toxicity, alcohol abuse, and hepatitis $\mathrm{C}$ are expected causal factors in this pathology, HIV viral proteins and antiretroviral therapy can also result in cholestatic liver disease (10) and hepatic fibrosis (11). Hepatitis C coinfection, which is associated with intravenous (iv) drug use (12), can worsen the damage (11).

In the NIH-Wide Strategic Plan Fiscal Years 2016-2020, the institute encourages researchers to study the interaction between diseases and conditions and their potential synergistic effects on the human body.

Corresponding Author: Michael Hambuchen Email: (hambuchen@marshall.edu); Department of Pharmaceutical Science and Research; Marshall University School of Pharmacy; 1 John Marshall Drive, Huntington, USA. 
The effects of METH are enhanced in rodent models of HIV (13-15), and the combination of HIV infection and METH use is associated with learning impairments and neurotoxicity (16). HIV is also known to have an effect on liver function in both humans infected with HIV (11) and in rodent models of HIV infection $(17,18)$. While the rodent HIV model studies show evidence for central nervous system based HIV-induced METH sensitization (1315), these studies do not account for any potential mediating effects of HIV-induced hepatic dysfunction. Additionally, there are no rodent studies demonstrating enhanced METH effects due to acetaminophen-, ethanol-, or hepatitis C-induced liver injury.

The current studies focused on hepatic dysfunction as it is a common feature of multiple comorbidities associated with METH use. Bile duct ligation (BDL) surgery was used to induce hepatic dysfunction in a preclinical rat model. The BDL surgery rapidly produces hepatic fibrosis (19) and is known to impair the clearance of hepatically eliminated drugs (20-22). In control animals, a sham surgery was performed which involved bile duct isolation without ligation. We hypothesized that BDL-induced hepatic dysfunction, independent of METH-induced toxicity, would both enhance METH-induced open field behavior and alter METH pharmacokinetics $(\mathrm{PK})$ resulting in increased serum and brain exposure to the drug.

\section{METHODS}

\section{Drugs, chemicals, and supplies}

(+)-Methamphetamine hydrochloride (METH) for administration was obtained from the National Institute on Drug Abuse. ( \pm )-METH and ( \pm )amphetamine (AMP) analytical standards in methanol and internal standard ( \pm )-1-phenyl-1,2dideutero-2-[trideuteromethyl]aminopropane $\left(\mathrm{METH}-\mathrm{D}_{5}\right)$ in methanol were purchased from Cerilliant (Round Rock, TX). All concentrations were calculated as free base. Isoflurane solution, injectable meloxicam $(5 \mathrm{mg} / \mathrm{mL})$ and gentamicin (40 $\mathrm{mg} / \mathrm{mL}$ ) were purchased from the University of Arkansas for Medical Sciences (UAMS) pharmacy supply room. Other reagents and supplies were purchased from Sigma Chemical Company or Thermo-Fisher Scientific, unless otherwise stated.

\section{Animals}

Male Wistar rats $(n=19)$ were purchased from Charles River Laboratories (Wilmington, MA) and arrived at the UAMS animal care facility at approximately 8 weeks old and $278 \pm 16 \mathrm{~g}$. Rats were dual housed in a $22^{\circ} \mathrm{C}$ room with a $14 / 10$-hour light/dark cycle, provided water ad libitum, and fed a sufficient amount ( $\sim 20 \mathrm{~g}$ daily) of standard rat chow to maintain a body weights less than $360 \mathrm{~g}$ over the experiment. Animal bodyweight, food consumption, appearance, and behavior were monitored daily under the guidance of a veterinarian. All experiments were conducted in accordance with the National Research Council Guide for Care and Use of Laboratory Animals, and the UAMS Institutional Animal Care and Use Committee.

\section{Animal experiment summary}

Sham and BDL surgeries were performed on day 0 . Serum was collected for alanine aminotransferase (ALT) determinations before surgery and 1, 4, 7, and 19 days afterward. PK studies were performed with $1 \mathrm{mg} / \mathrm{kg} \mathrm{sc}$ METH on days 7 and 19. Saline open field locomotor activity experiments were performed on days 9 and 10 . This was repeated with escalating sc METH doses $(0.3,1$, and $3 \mathrm{mg} / \mathrm{kg})$ on days 12,14 , and 16. On day 21, rats were euthanized under isoflurane anesthesia by decapitation two $\mathrm{h}$ after administration of a $1 \mathrm{mg} / \mathrm{kg}$ sc METH dose for collection of whole brains for determination of METH and AMP (metabolite) concentrations and liver slices for histopathological analysis. Detailed methods for each experiment are described below.

\section{BDL surgery}

Wistar rats received $1 \mathrm{mg} / \mathrm{kg}$ sc meloxicam and 2 $\mathrm{mg} / \mathrm{kg}$ sc gentamicin immediately before sham or BDL surgery in sterile conditions. Anesthesia was induced with 5\% inhaled isoflurane and maintained with $1-3 \%$ isoflurane as needed with an $\mathrm{O}_{2}$ flow rate of $1.5 \mathrm{~L} / \mathrm{min}$. The abdomen of each rat was shaved and aseptically prepared with chlorhexidine scrub and sterile saline. A 6-8 cm midline laparotomy was performed. The common bile duct was exposed by blunt dissection. In sham control animals, no sutures were placed and the bile duct was left intact. In the bile duct ligation rats, one ligature was placed with 3-0 silk proximal to the junction of the hepatic bile duct and the second was placed above the pancreatic duct entrance similar to the method of Yang et al. (23). Afterward, the ligatures were tightened, and the 
common bile duct was cut at the midpoint between the two ligatures. The peritoneal cavity was washed with warm sterile saline. The body wall was closed with absorbable suture material, and the skin was closed with stainless steel surgical clips. Each animal was monitored on a circulating heating pad until fully recovered. An autoclave was used to sterilize surgical instruments before surgery and a Germinator 500 (Roboz Surgical Instrument Co., Gaithersburg, MD) was used to sterilize the instruments between animal surgeries. Rats received post-operative analgesic (meloxicam $1 \mathrm{mg} / \mathrm{kg}$ ) and antibiotic (gentamicin $2 \mathrm{mg} / \mathrm{kg}$ ) for 3 days to minimize post-operative pain and risk of infection.

All sham rats $(n=6)$ completed the experimental protocol, but four rats in the BDL group were euthanized due to poor health (determined by a veterinarian) prior to the completion of the studies. The percentage of rats surviving after the BDL surgery in this study, however, was somewhat higher than in Yang et al. study (69 vs 58\%) (23).

\section{Open field behavior studies}

On experimental days, rats were placed into $60 \times 45$ x $70 \mathrm{~cm}$ open-top polyethylene chambers for one $\mathrm{h}$ prior to sc saline or METH administration. Animals remained in the chamber for six $\mathrm{h}$ after saline or $\operatorname{METH}(0.3,1,3 \mathrm{mg} / \mathrm{kg})$ administration.

Locomotor activity was measured as distance traveled $(\mathrm{cm})$ in four min bins using overhead mounted cameras interfaced with Ethovision 8 software (Noldus Information Technology, Inc., Sterling, VA). Duration of locomotor activity was determined similarly to a previous study (24) using the average saline value $+1 \mathrm{SD}$ value as baseline. After $3 \mathrm{mg} / \mathrm{kg}$ METH, the duration of stereotypy was reported as the duration of post-injection inactivity (less than animal baseline +1 standard deviation [SD]) prior to the delayed hyperlocomotor activity phase.

\section{PK and organ collection studies}

PK studies were performed before and after METHinduced open field behavior experiments. Collection time points were determined based on previous sc METH PK experiments in rats described by Gentry et al. (25). After $1 \mathrm{mg} / \mathrm{kg}$ METH was administered, $\sim 100 \mu \mathrm{l}$ blood was collected from the tail vein at 30 , 120,210 , and $300 \mathrm{~min}$. Rats were briefly anesthetized with isoflurane $(5 \%$ at $5 \mathrm{~L} / \mathrm{min}$ induction and $2.5 \%$ at $1.5 \mathrm{~L} / \mathrm{min}$ maintenance) for blood collection. Collected blood was allowed to clot, centrifuged for $7 \mathrm{~min}$ at 20,817 rcf for serum collection, and stored at $-80^{\circ} \mathrm{C}$.

For organ collection studies, rats were administered $1 \mathrm{mg} / \mathrm{kg} \mathrm{sc}$ METH. Two h later, the rats were decapitated under isoflurane anesthesia induced with $5 \%$ isoflurane at an $\mathrm{O}_{2}$ flow rate of 5 $\mathrm{L} / \mathrm{min}$. Prior to processing of brain and liver tissue, each brain, liver, heart, kidney, and spleen was weighed. Brain tissues, for the determination of METH and AMP concentrations, were quickly removed and flash frozen in liquid nitrogen prior to storage at $-80^{\circ} \mathrm{C}$. Liver samples, for histology, were sliced into small rectangular sections and stored in formalin.

\section{METH and AMP quantitation}

Prior to extraction, whole brains were thawed on ice, diluted with $4 \mathrm{~mL}$ water per $\mathrm{g}$ of tissue, and thoroughly homogenized. A previously described extraction method for $(3,4)$ methylenedioxypyrovalerone quantitation (26) was adapted for the extraction of METH and AMP from serum and brain tissue. Differences include the use of METH- $\mathrm{D}_{5}$ internal standard in $0.1 \%$ formic acid in acetonitrile, the adjustment of all volumes proportionally to the increase from 20 to $25 \mu 1$ sample aliquot volume, and the use of $75 \mu 1$ of $0.1 \%$ formic acid for reconstitution prior to injection.

The extracted sample $(65 \mu \mathrm{l})$ was transferred to an autoinjector plate and $7.5 \mu 1$ was injected onto an Acquity UPLC BEH C18 $1.7 \mu \mathrm{m}$ (2.1 i.d. x $100 \mathrm{~mm}$ ) column (Waters Corp, Milford, MA). The liquid chromatography tandem mass spectrometry (LC/MS-MS) system consisted of an Acquity Ultra Performance Liquid Chromatography system interfaced to a Quattro Premier XE mass spectrometer (Waters Corp) with an electrospray ion source operated in the positive ion mode.

Analysis of METH and AMP concentrations in extracted samples was performed by a previously described LC/MS-MS method (27). The lower and upper limit of quantification for METH and AMP was 1 and $1000 \mathrm{ng} / \mathrm{mL}$, respectively. All predicted values for calibration and quality control standards were within $\pm 15 \%$ of the nominal value (within \pm $20 \%$ for the LLOQ).

\section{PK analysis}

Serum METH and AMP concentration time curves were analyzed by noncompartmental analysis 
(accounting for extravascular sc administration) using WinNonlin (V6.4, Certara USA, Princeton, $\mathrm{NJ}$ ). PK parameters including area under the concentration time curve from time 0 to infinity $\left(\mathrm{AUC}_{0-\infty}\right)$, terminal elimination half-life $\left(\mathrm{t}_{1 / 2}\right)$, estimated clearance $(\mathrm{Cl})$, and estimated volume of distribution $(\mathrm{Vd})$ were determined for METH in each rat. The $t_{1 / 2}$ values were determined using the slope of the last three serum METH concentration values. The $\mathrm{Cl}$ and $\mathrm{Vd}$ values were considered estimates since $\mathrm{AUC}_{0-\infty}$ was not determined after an iv dose in the current study to determine bioavailability.

Because of insufficient sampling to determine

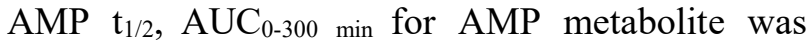
determined and used to calculate the ratio of AMP/METH $\mathrm{AUC}_{0-300 \mathrm{~min}}$. All PK parameters for sham and BDL rats are reported as mean $\pm \mathrm{SD}$ except for $t_{1 / 2}$ which was reported as harmonic mean \pm pseudo standard deviation (28).

\section{Histology and ALT analysis}

Serum samples for ALT determination were collected before sham or BDL surgery and 1, 4, 7, and 19 days after surgery. Day 7 samples were collected just before the first PK study and day 19 samples were collected just before the second PK study. Samples were collected as described in the PK and terminal organ collection studies section, but were clotted on ice and centrifuged at $4^{\circ} \mathrm{C}$. ALT in thawed serum was determined with an ALT (SGPT) Liquid Reagent Kit (Pointe Scientific, Canton, MI) at $37^{\circ} \mathrm{C}$ and $340 \mathrm{~nm}$ absorbance.

For histology, a portion of each liver was fixed in $10 \%$ phosphate-buffered formalin. The fixed tissues were then embedded in paraffin wax and 5 $\mu \mathrm{m}$ sections were cut and dried on glass slides for staining with hematoxylin and eosin (H\&E). Images were collected using a Nikon Eclipse microscope. For each animal, bile duct-like structures were counted in 4 to 5 random high power $(400 \mathrm{x})$ fields (HPF). The number of bile duct-like structures per field was averaged for each animal, and the means of those averages were calculated for each group.

\section{STATISTICAL ANALYSIS}

Results from the open field locomotor activity experiments were analyzed using a two-factor mixed-model analysis of variance (ANOVA) with sham or BDL surgery as the between-subjects factor and dose as the within-subjects factor. Results from the PK and ALT experiments were analyzed using a two-factor mixed-model analysis of variance (ANOVA) with sham or BDL surgery as the between-subjects factor and experimental day as the within-subjects factor. Following a statistically significant ANOVA, Holm-Sidak multiple comparisons tests (or simple main effects tests in the case of a statistically significant interaction) were performed for all pairwise comparisons. Histology, weight percentage of baseline AUC, organ weight, brain concentration, and duration of $3 \mathrm{mg} / \mathrm{kg} \mathrm{METH}$ induced stereotypy duration were initially analyzed for equal variances using an F-test. If an F-test revealed a statistically significant inequality between variances, an unpaired Welch's t-test was performed. In cases of equal variances, data were analyzed using an unpaired t-test. To assess the magnitude of the association between brain METH concentrations and the duration of locomotor activity after $1 \mathrm{mg} / \mathrm{kg} \mathrm{sc}$ METH, a Pearson's $r$ correlation analysis was performed on the pooled (sham and BDL) data. Linear regression analysis was used to fit this data. Statistical analysis and creation of figures was performed with GraphPad Prism software (V7, La Jolla, CA, USA). Statistical significance was declared at $p<.05$. The detailed results of all statistical analyses are included in the supplemental section.

\section{RESULTS}

Holm-Sidak simple main effects tests were performed when the two-factor mixed-model ANOVA determined an interaction. Key findings of the Holm-Sidak simple main effects tests and t-tests are either marked in the corresponding figure or in the results. Detailed statistical comparisons for these and other experiments are included in the supplemental file.

\section{BDL-induced liver injury effects}

Figure 1 shows the serum ALT values before surgery (Pre); after surgery, but before METH (days $1-7$ ), and after multiple METH administrations (day 19). For these values, there was a significant interaction between intervention (sham vs BDL) and time [F(4, $52)=58.79, p<.0001]$. Figure 2 shows (A) representative liver slices and (B) bile duct proliferation. 


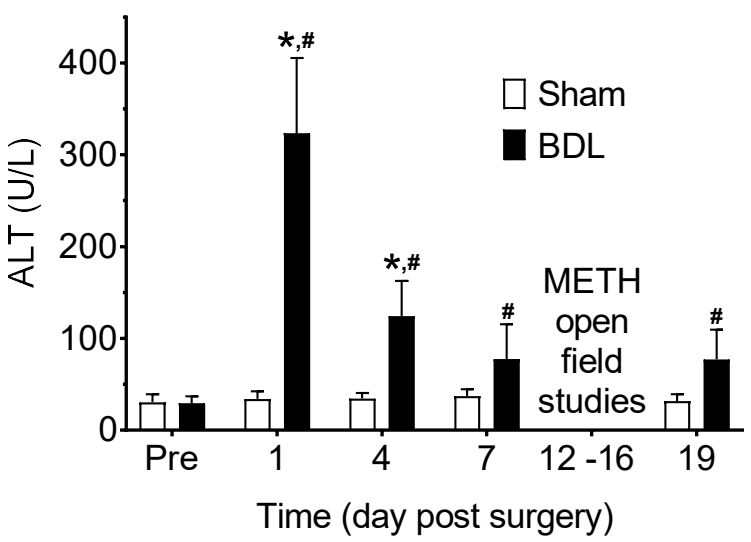

Figure 1. ALT on days after surgery. Mean ALT + SD was stable in the sham group on all measurement days. There was a significant elevation on days 1 and 4 in the BDL compared to sham rats. Day 7 levels were measured before any exposure to METH. ALT did not increase in sham or BDL rats after METH exposure. The * denotes significant difference between sham and BDL groups $(\mathrm{p}<$ $.05)$. The \# denotes significant difference between the presurgery (Pre) and post-surgery values in the BDL group ( $\mathrm{p}$ $<.05)$. $(\mathrm{n}=6$ sham, $\mathrm{n}=9$ BDL $)$.

\section{Hepatic dysfunction effects on METH-induced open field behavior}

Figure 3 shows the Saline and 0.3, 1, and $3 \mathrm{mg} / \mathrm{kg}$ METH-induced locomotor activity (measured as distance traveled in 4 min intervals) over time in min in sham compared to BDL rats. Figure 4 includes METH-induced (A) total distance traveled, (B) duration of activity, and (C) duration of stereotypy.
For the total distance traveled values (Figure 4A), there was a significant interaction between intervention (sham vs BDL) and time $[F(2,26)=$ $3.531, p=.044]$. For the duration of locomotor activity values (Figure 4B), there was a significant interaction between intervention (sham vs BDL) and time $[F(2,26)=10.86, p=.0004]$. In addition, the simple main effects test showed differences in activity duration between all doses within each group. Figure 4C shows duration of stereotypy. Note that stereotypy was not detected in sham or BDL rats after $0.3 \mathrm{mg} / \mathrm{kg}$ METH and in sham rats after 1 $\mathrm{mg} / \mathrm{kg}$ METH. One h of stereotypy was observed in a single BDL rat after $1 \mathrm{mg} / \mathrm{kg}$ METH (not shown in figure).

\section{Hepatic dysfunction effects on METH and AMP $P K$ and brain disposition}

Figure 5 shows serum METH and AMP (METH metabolite) concentrations over time, and the PK parameters in Table 1 were generated from these data. There was a significant main effect of intervention (sham vs BDL) for $\mathrm{AUC}_{0-\infty}[F(1,13)=$ 25.37, $p=.0002]$, estimated $\mathrm{Cl}[F(1,13)=31.5, p<$ $.0001]$, and percent ratio serum AMP:METH AUC 0 $300[F(1,13)=7.942, p=.0145]$. For $\mathrm{t}_{1 / 2}$, there was a significant interaction between intervention (sham vs BDL) and time $[F(1,13)=7.1, p=.0195]$. For $\mathrm{Vd}$, there was a significant interaction between intervention (sham vs BDL) and time $[F(1,13)=$ 7.758, $p=.0155]$.
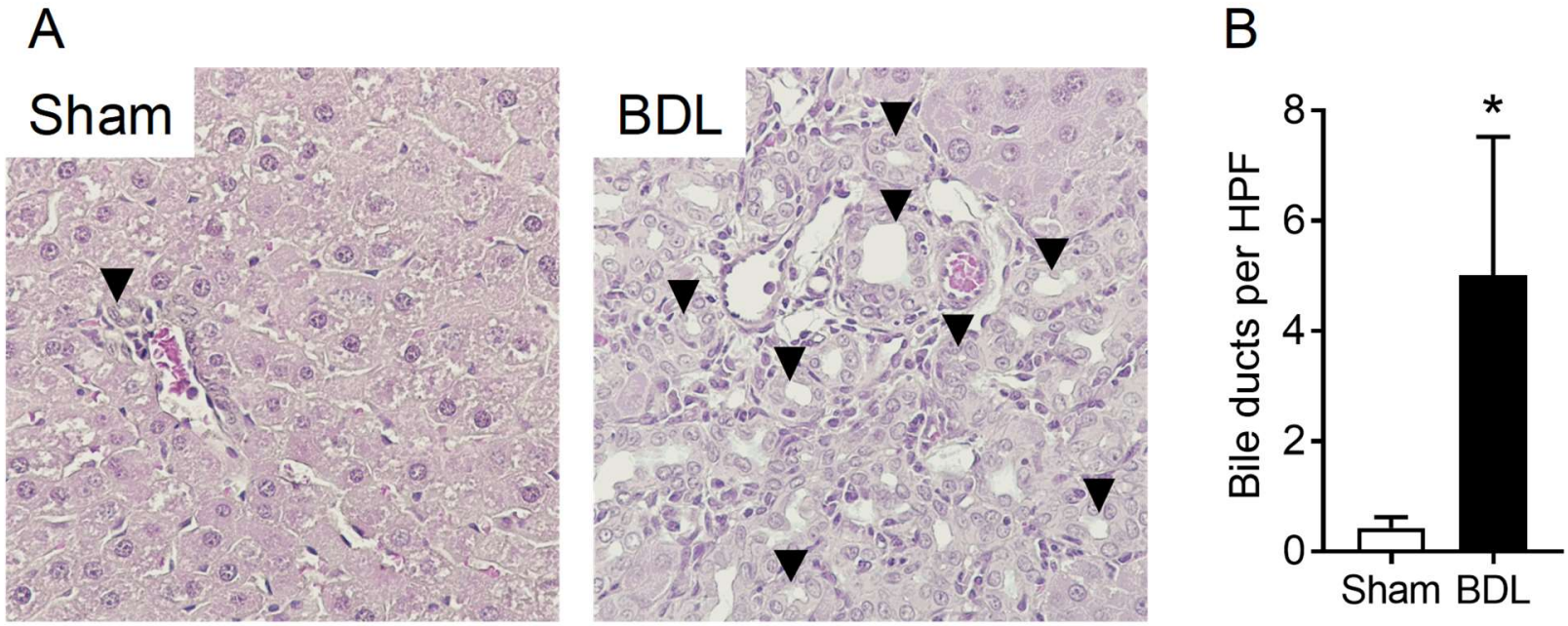

Figure 2. Histopathology of BDL. Liver tissue was harvested 21 days after sham or BDL surgery. Tissue was analyzed for (A) H\&E staining and (B) quantification of bile ducts per high power field (HPF). Arrowheads point to examples of bile ductlike structures. Data are expressed as mean $\pm \mathrm{SD}$ for $\mathrm{n}=5$. The $*$ denotes significant difference between sham and BDL groups $(\mathrm{p}<.05)$. Supplemental table S1 shows day 21 organ weights, and Figure S1 shows the percent body weight change over time. 

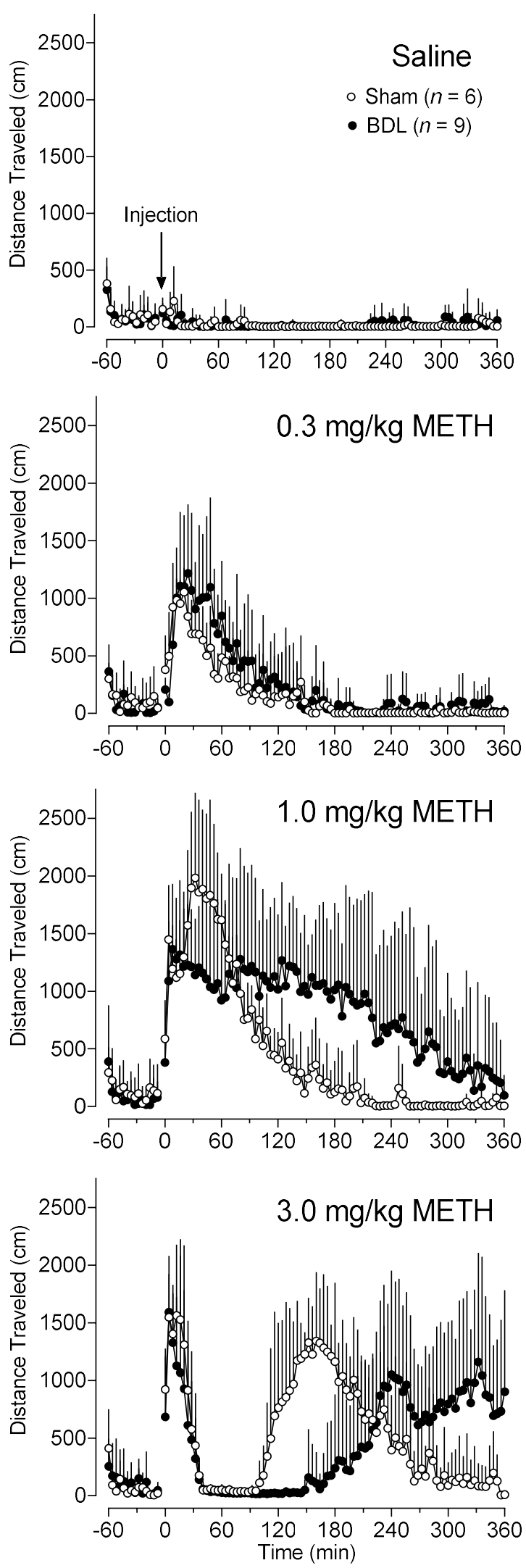

Figure 3. Distance traveled over time after saline and $(0.3,1$, and $3 \mathrm{mg} / \mathrm{kg}) \mathrm{METH}$ administration. Data reported as average activity + SD in 4 min bins in sham and BDL groups. Saline or METH was injected at 0 min. $(n=6$ sham, $n=9$ BDL $)$. 

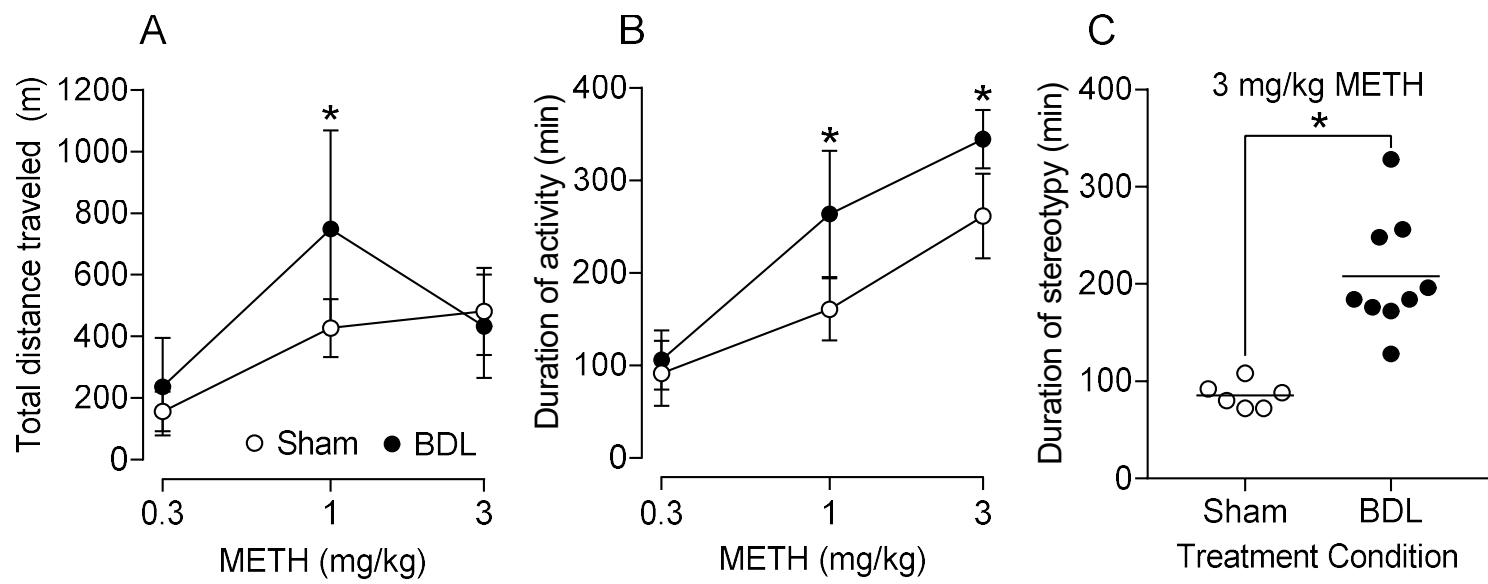

Figure 4. Open field behavior after METH administration. Mean \pm SD total distance traveled in $6 \mathrm{~h}$ after $0.3,1$, and $3 \mathrm{mg} / \mathrm{kg}$ METH (A), duration of locomotor activity after $0.3,1$, and $3 \mathrm{mg} / \mathrm{kg}$ METH (B), and duration of stereotypy episode after 3 $\mathrm{mg} / \mathrm{kg}$ METH $(\mathrm{C})$ were compared between sham and BDL groups. The * denotes significant difference between sham and BDL groups $(\mathrm{p}<.05)$. $(\mathrm{n}=6$ sham, $\mathrm{n}=9 \mathrm{BDL})$.
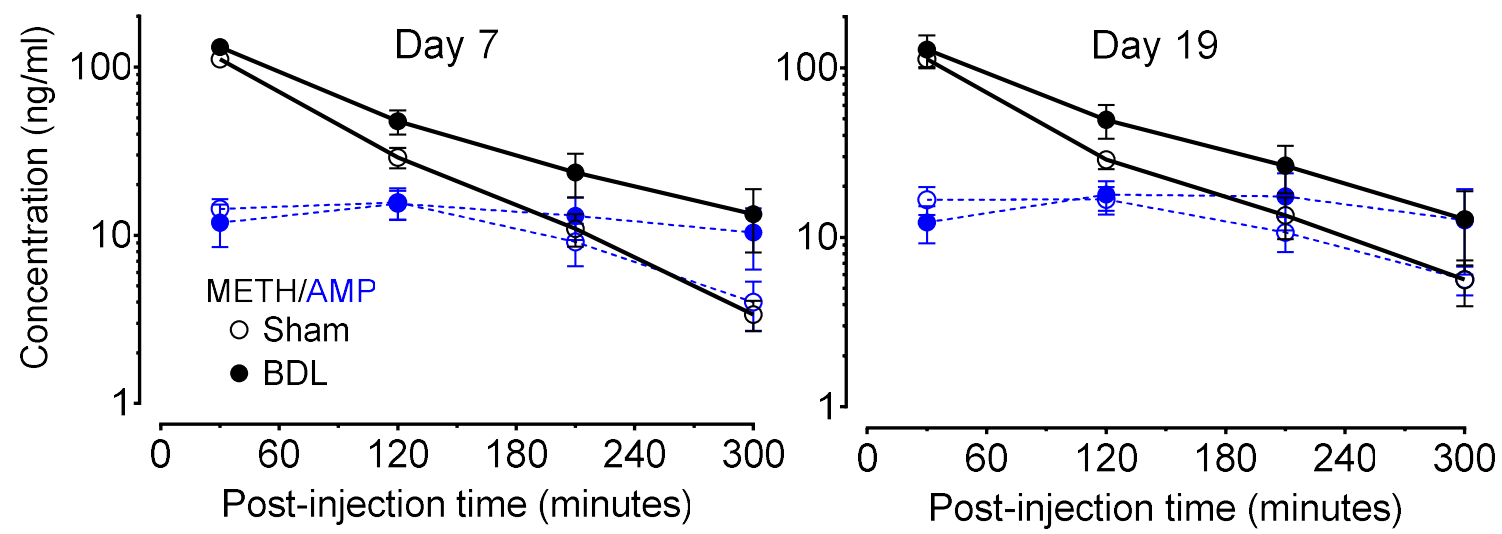

Figure 5. METH and AMP Serum concentration after $1 \mathrm{mg} / \mathrm{kg}$ METH administration before (day 7) and after (day 19) open field experiments. Mean serum METH (black solid lines) and amphetamine (AMP) metabolite (blue dashed lines) concentrations \pm SD are plotted for the sham (open circles) and BDL (closed circles) groups on day 7 (METH naïve) and day 19 (post-METH exposure). ( $n=6$ sham, $n=9$ BDL).

Table 1. Mean METH PK parameters \pm SD after $1 \mathrm{mg} / \mathrm{kg}$ SC METH.

\begin{tabular}{|c|c|c|c|c|}
\hline & \multicolumn{2}{|c|}{ Day 7 (METH naive) } & \multicolumn{2}{|c|}{ Day 19 (post-METH exposure) } \\
\hline & Sham $(n=6)$ & $\operatorname{BDL}(n=9)$ & Sham $(n=6)$ & $\operatorname{BDL}(n=9)$ \\
\hline $\begin{array}{l}\text { METH AUC } 0-\infty \\
\text { (ng } \mathrm{min} / \mathrm{mL} \text { ) }\end{array}$ & $10744 \pm 883$ & $16965 \pm 2831^{\mathrm{a}}$ & $11537 \pm 889$ & $16918 \pm 4372^{a}$ \\
\hline $\begin{array}{l}\text { METH } t_{1 / 2} \\
(\min )^{b}\end{array}$ & $58 \pm 5^{\mathrm{c}}$ & $94 \pm 17^{a}$ & $75 \pm 18$ & $87 \pm 15$ \\
\hline $\begin{array}{l}\text { METH Cl } \\
(\mathrm{mL} / \mathrm{min} \mathrm{kg})^{\mathrm{d}}\end{array}$ & $94 \pm 7$ & $60 \pm 9^{\mathrm{a}}$ & $87 \pm 7$ & $64 \pm 20^{\mathrm{a}}$ \\
\hline $\begin{array}{l}\text { METH Vd } \\
(\mathrm{mL} / \mathrm{kg})^{\mathrm{d}}\end{array}$ & $7819 \pm 798^{c}$ & $8366 \pm 1144$ & $9939 \pm 2424$ & $7881 \pm 869^{\mathrm{a}}$ \\
\hline AMP/METH AUC ${ }_{0-300}(\%)$ & $31 \pm 4$ & $25 \pm 5^{\mathrm{a}}$ & $32 \pm 4$ & $27 \pm 4^{\mathrm{a}}$ \\
\hline
\end{tabular}

a) significant difference between bile duct ligation (BDL) and sham groups $(\mathrm{p}<.05)$

b) $t_{1 / 2}$ values reported as harmonic mean \pm pseudo-standard deviation (28)

c) significant differences between day 7 and $19(\mathrm{p}<.05)$

d) estimated values due to sc rather than iv administration 
The day 21, two hours post- $1 \mathrm{mg} / \mathrm{kg}$ sc METH brain concentrations were significantly $(\mathrm{p}<.05)$ elevated in BDL $(501 \pm 127 \mathrm{ng} / \mathrm{g})$ compared to sham $(272 \pm 51 \mathrm{ng} / \mathrm{g})$ rats. The AMP brain concentrations in BDL $(234 \pm 65 \mathrm{ng} / \mathrm{g})$ and sham $(190 \pm 38 \mathrm{ng} / \mathrm{g})$ were not significantly different. The percent AMP to METH ratio in the brain was significantly $(\mathrm{p}<.05)$ reduced in the BDL $(47 \pm 10 \%)$ compared to sham $(70 \pm 9 \%)$ rats. Figure 6 shows the correlation between duration of locomotor activity and brain METH concentrations after $1 \mathrm{mg} / \mathrm{kg}$ sc METH.



Figure 6. Correlation between duration of locomotor activity and brain METH concentrations after $1 \mathrm{mg} / \mathrm{kg}$ METH administration. Each sham (open circles) and BDL (closed circles) rat is plotted. The best-fit line equation was $\mathrm{y}=0.47 \mathrm{x}+30$.

\section{DISCUSSION}

The BDL surgery-induced liver dysfunction was measured in several ways. ALT, a liver enzyme that is released by liver damage and is a standard clinical laboratory test for liver injury, was found to be significantly elevated in BDL compared to sham rats on day 1 and 4 after surgery (Figure 1). While this biomarker recovered to some degree, it remained significantly elevated compared to pre-METH baseline values at all time points in the BDL rats. This was consistent with what is known about the time course of BDL-induced liver disease in rodents. Others have reported that biliary infarcts develop, with passive release of ALT from necrotic hepatocytes, in the first 24-72 h post-BDL (29). At later time points, compensatory and reparative processes begin and the initial acute injury resolves. However, this is followed by a long period of chronic liver disease characterized by elevated bilirubin and bile duct proliferation. Indeed, bile duct proliferation was evident in our BDL animals on experimental day 21 by histopathology (Figure 2), and liver mass was increased almost 2-fold compared to sham livers (Table S1). Hepatomegaly is also common clinically with HIV and hepatitis coinfection (30). A relevant qualitative observation of the BDL-pathology was the bright yellow color of the urine (bilirubinuria) and serum (bilirubinemia) due to bilirubin elevation in BDL animals that was not visible in the sham animals. These data show that the damage induced by the BDL surgery persisted for the entirety of the study.

In addition, spleen weight was significantly increased in the BDL group compared to the sham group (Table S1). This was not surprising as splenomegaly is associated with liver disease (31). While average rat weight in the BDL group returned to the pre-surgery baseline by approximately day 7 , the expected increase in rat weight lagged behind that of the sham group (Figure S1). The increase over time was, however, parallel between the two groups.

The hepatic dysfunction resulted in augmented METH-induced behavior relative to sham control rats. While open field locomotor activity data for both sham and BDL rats after $0.3 \mathrm{mg} / \mathrm{kg}$ METH were similar, the patterns of activity in BDL rats after 1 and $3 \mathrm{mg} / \mathrm{kg}$ METH were considerably different from the patterns of activity in sham rats (Figure 3). The similar locomotor responses between groups to the $0.3 \mathrm{mg} / \mathrm{kg}$ METH may be due to the contributions of the saturable renal component of METH elimination (32). Considering total distance traveled, it appears that the METH enhancing effect of hepatic dysfunction decreased with the $3 \mathrm{mg} / \mathrm{kg}$ METH dose (Figure 4A). This is due to a dose dependent increase in the duration of the stereotypy phase of activity (33). At higher doses, METH-induced open field behavior becomes multiphasic; the stereotypy initially suppresses horizontal movement and is followed by a post-stereotypy elevated horizontal movement phase (34). This was observed on average in the period from approximately $30 \mathrm{~min}$ after the 3 $\mathrm{mg} / \mathrm{kg}$ dose until 90 and $150 \mathrm{~min}$ in the sham and BDL groups, respectively (Figure 3). Also in the current study, the total distance traveled data at 3 $\mathrm{mg} / \mathrm{kg}$ was misleading as many of the BDL rats were still active at the $6 \mathrm{~h}$ experiment cutoff (Figure 3). Therefore, the duration of METH-induced locomotor activity is a more useful measurement for 
determining differences in high-dose METH effects produced by a condition or treatment. Indeed, there were significant increases in the duration of METHinduced locomotion compared to sham animals following administration of 1 and $3 \mathrm{mg} / \mathrm{kg}$ (Figure 4B). At $3 \mathrm{mg} / \mathrm{kg}$, duration of stereotypy was significantly increased in BDL rats (Figure 4C). One particularly sensitive animal in the BDL group remained in stereotypy for the entire $6 \mathrm{~h}$ period.

To investigate contributions of serum PK to the BDL enhancement of METH-induced activity, a 1 $\mathrm{mg} / \mathrm{kg}$ METH PK study was performed before and after METH exposure on day 7 and 19, respectively, after sham or BDL surgery (Figure 5). The rat serum METH concentrations were in the range of the concentrations measured in humans after a $0.5 \mathrm{mg} / \mathrm{kg}$ iv METH dose (35). Note that this human dose is within the range of typical recreational doses (36), and produces significant intoxication in humans (35). The value for the $\mathrm{AUC}_{0-\infty}$ was substantially higher, estimated $\mathrm{Cl}$ was significantly lower, and $\mathrm{t}_{1 / 2}$ was significantly longer in the BDL compared to sham rats (Table 1) on day 7 . These data suggest greater serum METH exposure in the BDL group. The significant reduction in estimated $\mathrm{Cl}$ on both days was possibly due to reduced metabolism (see the reduction in AMP metabolite production in the BDL compared to sham rats). This, however, does not account for any potential changes in renal metabolism.

On day 19, after exposure to four METH doses, the values for METH $\mathrm{AUC}_{0-\infty}$, percent $\mathrm{AMP}$ to METH $\mathrm{AUC}_{0-300}$ min, and estimated $\mathrm{Cl}$ were still significantly different in the BDL compared to the sham group (Table 1). The PK parameters were stable over time and after multiple METH administrations in the BDL group. There were, however, significant increases in METH estimated Vd between day 7 and 19 in the sham group. Since $\mathrm{t}_{1 / 2}$ is dependent on both $\mathrm{Vd}$ and $\mathrm{Cl}$, $\left(\mathrm{t}_{1 / 2}\right.$ $=0.693 \times \mathrm{Vd} / \mathrm{Cl})$, the significantly increased $\mathrm{Vd}(27 \%$ increase) and somewhat decreased $\mathrm{Cl}$ (7\% decrease) values on day 19 compared to day 7 resulted in a significantly prolonged $t_{1 / 2}$ in the sham group on day 19 compared to day 7. This caused, on day 19, the estimated METH Vd to be different between the BDL and sham groups and the METH $t_{1 / 2}$ to no longer be different between the two groups. The decrease in $\mathrm{Cl}$ and increase in $\mathrm{t}_{1 / 2}$ is expected as repeated METH administration in healthy rats is known to result in reduced METH elimination and a longer $\mathrm{t}_{1 / 2}$ through changes in renal elimination (37). The METH Vd did not increase in the previous study in the METH-sensitized compared to METH-naïve rats, but in that study these groups were age matched. In our study, it is possible that the change in $\mathrm{Vd}$ in sham rats over time was due to a change in protein binding or total body fat. Regardless, the key parameter, $\mathrm{AUC}_{0-\infty}$ (a measure of serum $\mathrm{METH}$ exposure), did not significantly change over time in the sham group. Most importantly, $\mathrm{AUC}_{0-\infty}$ was significantly elevated in the BDL compared to sham group on both day 7 and 19 which showed that hepatic dysfunction led to increased serum METH exposure during the study period. Considering that BDL group parameters were not affected by the repeated METH administration, future studies may be needed to assess unchanged renal clearance of METH in the urine of control and BDL rats.

Although different rat strains (Wistar vs. Sprague Dawley), collection methods (tail vein vs. catheter), and numbers of serum collection time points (4 vs.10) were used, the PK data from the METH naïve sham rats (day 7) in this study were very consistent with PK data determined by Gentry et al. after sc METH administration (25). For example, the METH $\mathrm{AUC}_{0-\infty}$ of $10744 \pm 883 \mathrm{ng}$ $\mathrm{min} / \mathrm{mL}$ and $\mathrm{Cl}$ of $94 \pm 7 \mathrm{~mL} / \mathrm{min} \mathrm{kg}$ were similar to the $10666 \pm 1805 \mathrm{ng} \mathrm{min} / \mathrm{mL}$ (dose adjusted) and 94 $\pm 17 \mathrm{~mL} / \mathrm{min} \mathrm{kg}$ values in the previous study. In addition, Gentry reported a sc bioavailability of $100 \%$ for METH in Sprague Dawley rats. This increases the probability that the estimated $\mathrm{Cl}$ and $\mathrm{Vd}$ values in the current sc study are accurate.

The reduction in METH clearance resulted in greater brain exposure to METH in the BDL group. Two hours after $1 \mathrm{mg} / \mathrm{kg} \mathrm{sc}$ METH on day 21, brain concentrations of METH were significantly elevated by $84 \%$ in the BDL compared to sham rats $(\mathrm{p}<.05)$. In addition, the percent AMP:METH ratio in the brain was significantly reduced in the BDL compared to sham rats $(\mathrm{p}<.05)$. The finding that BDL-induced increases in METH brain concentrations were associated with increased activity measures (Fig 6) was not surprising since the treatment of rats with anti-METH monoclonal antibodies or METH vaccines produces decreases in brain METH concentrations that are associated with decreases in METH-induced behaviors $(38,39)$. While the change in PK values was likely responsible for the BDL-induced increase in brain METH concentrations, circulating bilirubin due to 
BDL surgery is associated with increased permeability of the blood brain barrier (40) which could also increase brain METH concentrations.

One limitation of this study is the severity of the liver pathology produced by the BDL surgery (19). Future studies would need to modify the BDLsurgical technique to produce a range of hepatic dysfunction and study additional hepatotoxicity biomarkers to determine and characterize potential liver damage due to METH exposure. Additionally, this study was only performed in male rats, but there are known sex differences in METH-induced rat behavior and METH PK $(32,41-43)$ and in effects on human behavior and brain function (44).

\section{CONCLUSION}

BDL-induced liver damage both substantially reduced METH clearance from the serum and substantially increased METH concentrations in the brain. While $0.3 \mathrm{mg} / \mathrm{kg}$ METH-induced locomotor activity was not increased by hepatic dysfunction, the 1 and $3 \mathrm{mg} / \mathrm{kg}$ METH-induced activity was significantly augmented. The enhancement of METH effects by comorbid conditions that result in hepatic dysfunction may serve as a barrier to successful METH use disorder pharmacotherapy. With additional development, this model could be used to test pharmacotherapies for METH-use disorder and make preclinical predictions of their effectiveness and/or toxicity in vulnerable populations suffering from both METH use disorder and comorbid hepatic dysfunction-inducing conditions.

\section{CONFLICT OF INTEREST}

S. Michael Owens is Chief Scientific Officer of and has financial interests in InterveXion Therapeutics, LLC, a pharmaceutical biotech company, whose main interest is the development of antibody medications for the treatment of human diseases, including drug abuse.

\section{ACKNOWLEDGMENTS}

This work was supported by the Wilbur D. Mills Endowed Chair in Alcohol and Substance Abuse Prevention and NIH/NIDA T32DA022981. In addition, the authors thank Mike West, Samantha
McClenahan, and Chris Bolden for technical assistance.

\section{ABBREVIATIONS}

ALT, alanine aminotransferase; AMP, amphetamine; AUC, area under the concentration time curve; $\mathrm{BDL}$, bile duct ligation; $\mathrm{Cl}$, estimated clearance; METH, methamphetamine; PK, pharmacokinetics; $\mathrm{t}_{1 / 2}$, half-life; $\mathrm{Vd}$, estimated volume of distribution.

\section{REFERENCES}

1. DEA. National Drug Threat Assessment. In: Administration USDoJaDE, editor. 2018.

2. Cook R, Quinn B, Heinzerling K, Shoptaw S. Dropout in clinical trials of pharmacological treatment for methamphetamine dependence: the role of initial abstinence. Addiction. 2017;112(6):107785. doi: 10.1111/add.13765

3. Owens SM, Atchley WT, Hambuchen MD, Peterson EC, Gentry WB. Monoclonal antibodies as pharmacokinetic antagonists for the treatment of $(+)$ methamphetamine addiction. CNS Neurol Disord Drug Targets. 2011;10(8):892-8. doi: 10.2174/187152711799219370

4. Beardsley PM, Hauser KF. Glial modulators as potential treatments of psychostimulant abuse. Adv Pharmacol. 2014;69:1-69. doi: 10.1016/B978-0-12420118-7.00001-9

5. Stevens MW, Henry RL, Owens SM, Schutz R, Gentry WB. First human study of a chimeric antimethamphetamine monoclonal antibody in healthy volunteers. MAbs. 2014;6(6):1649-56. doi: 10.4161/19420862.2014.976431

6. Worley MJ, Heinzerling KG, Roche DJ, Shoptaw S. Ibudilast attenuates subjective effects of methamphetamine in a placebo-controlled inpatient study. Drug Alcohol Depend. 2016;162:245-50. doi: 10.1016/j.drugalcdep.2016.02.036

7. Hartzler B, Dombrowski JC, Crane HM, Eron JJ, Geng EH, Christopher Mathews W, et al. Prevalence and Predictors of Substance Use Disorders Among HIV Care Enrollees in the United States. AIDS Behav. 2017;21(4):1138-48. doi: 10.1007/s10461016-1584-6

8. Gonzales R, Marinelli-Casey P, Shoptaw S, Ang A, Rawson RA. Hepatitis $\mathrm{C}$ virus infection among methamphetamine-dependent individuals in outpatient treatment. J Subst Abuse Treat. 2006;31(2):195-202. doi: 10.1016/j.jsat.2006.04.006

9. Havens JR, Stoops WW, Leukefeld CG, Garrity TF, Carlson RG, Falck R, et al. Prescription opiate misuse among rural stimulant users in a multistate 
community-based study. Am J Drug Alcohol Abuse. 2009;35(1):18-23. doi: 10.1080/00952990802326298

10. Te HS. Cholestasis in HIV-infected patients. Clin Liver Dis. 2004;8(1):213-28, viii-ix. doi: 10.1016/S1089-3261(03)00129-6

11. Anadol E, Lust K, Boesecke C, Schwarze-Zander C, Mohr R, Wasmuth JC, et al. Exposure to previous cART is associated with significant liver fibrosis and cirrhosis in human immunodeficiency virus-infected patients. PLoS One. 2018;13(1):e0191118. doi: 10.1371/journal.pone.0191118

12. Bosh KA, Coyle JR, Hansen V, Kim EM, Speers S, Comer M, et al. HIV and viral hepatitis coinfection analysis using surveillance data from 15 US states and two cities. Epidemiol Infect. 2018;146(7):92030. doi: $10.1017 / \mathrm{S} 0950268818000766$

13. Liu X, Chang L, Vigorito M, Kass M, Li H, Chang SL. Methamphetamine-induced behavioral sensitization is enhanced in the HIV-1 transgenic rat. J Neuroimmune Pharmacol. 2009;4(3):309-16. doi: 10.1007/s11481-009-9160-8

14. Kass MD, Liu X, Vigorito M, Chang L, Chang SL. Methamphetamine-induced behavioral and physiological effects in adolescent and adult HIV-1 transgenic rats. J Neuroimmune Pharmacol. 2010;5(4):566-73. doi: 10.1007/s11481-010-9221-z

15. Kesby JP, Najera JA, Romoli B, Fang Y, Basova L, Birmingham A, et al. HIV-1 TAT protein enhances sensitization to methamphetamine by affecting dopaminergic function. Brain Behav Immun. 2017;65:210-21. doi: 10.1016/j.bbi.2017.05.004

16. Soontornniyomkij V, Kesby JP, Morgan EE, Bischoff-Grethe A, Minassian A, Brown GG, et al. Effects of HIV and Methamphetamine on Brain and Behavior: Evidence from Human Studies and Animal Models. J Neuroimmune Pharmacol. 2016;11(3):495-510. doi: 10.1007/s11481-016-96990

17. Agarwal N, Iyer D, Gabbi C, Saha P, Patel SG, Mo $\mathrm{Q}$, et al. HIV-1 viral protein $\mathrm{R}(\mathrm{Vpr})$ induces fatty liver in mice via LXRalpha and PPARalpha dysregulation: implications for HIV-specific pathogenesis of NAFLD. Sci Rep. 2017;7(1):13362. doi: 10.1038/s41598-017-13835-w

18. Ghoneim RH, Piquette-Miller M. EndotoxinMediated Downregulation of Hepatic Drug Transporters in HIV-1 Transgenic Rats. Drug Metab Dispos. 2016;44(5):709-19. doi: 10.1124/dmd.115.067827

19. Tarcin O, Basaranoglu M, Tahan V, Tahan G, Sucullu I, Yilmaz N, et al. Time course of collagen peak in bile duct-ligated rats. BMC Gastroenterol. 2011;11:45. doi: 10.1186/1471-230X-11-45

20. Basseches PJ, DiGregorio GJ. Pharmacokinetics of procainamide in rats with extrahepatic biliary obstruction. J Pharm Sci. 1982;71(11):1256-9. doi: 10.1002/jps.2600711118

21. Esmaeili Z, Mohammadi S, Nezami A, Rouini MR, Ardakani YH, Lavasani H, et al. A disposition kinetic study of Tramadol in bile duct ligated rats in perfused rat liver model. Biomed Pharmacother. 2017;91:2516. doi: 10.1016/j.biopha.2017.04.082

22. Han SS, Kim KY, Ham SH, Sohn DH, Kim JB. Pharmacokinetics of theophylline: effects of hepatic fibrosis in rats induced bile duct ligation. Biol Pharm Bull. 1995;18(3):470-3. doi: 10.1248/bpb.18.470

23. Yang Y, Chen B, Chen Y, Zu B, Yi B, Lu K. A comparison of two common bile duct ligation methods to establish hepatopulmonary syndrome animal models. Lab Anim. 2015;49(1):71-9. doi: $10.1177 / 0023677214558701$

24. Byrnes-Blake KA, Laurenzana EM, Carroll FI, Abraham P, Gentry WB, Landes RD, et al. Pharmacodynamic mechanisms of monoclonal antibody-based antagonism of $(+)$-methamphetamine in rats. Eur J Pharmacol. 2003;461(2-3):119-28. doi: 10.1016/S0014-2999(03)01313-X

25. Gentry WB, Ghafoor AU, Wessinger WD, Laurenzana EM, Hendrickson HP, Owens SM. (+)Methamphetamine-induced spontaneous behavior in rats depends on route of (+)METH administration. Pharmacol Biochem Behav. 2004;79(4):751-60. doi: 10.1016/j.pbb.2004.10.006

26. Hambuchen MD, Hendrickson HP, Owens SM. Chiral determination of 3,4methylenedioxypyrovalerone enantiomers in rat serum. Anal Methods. 2017;9(4):609-17. doi: 10.1039/C6AY03176E

27. Hambuchen MD, Carroll FI, Ruedi-Bettschen D, Hendrickson HP, Hennings LJ, Blough BE, et al. Combining Active Immunization with Monoclonal Antibody Therapy To Facilitate Early Initiation of a Long-Acting Anti-Methamphetamine Antibody Response. J Med Chem. 2015;58(11):4665-77. doi: 10.1021/acs.jmedchem.5b00220

28. Lam FC, Hung CT, Perrier DG. Estimation of variance for harmonic mean half-lives. J Pharm Sci. 1985;74(2):229-31. doi: 10.1002/jps.2600740229

29. Harnoss JC, Heidecke CD, Vollmar B, Eipel C. In vivo imaging of bile accumulation and biliary infarction after common bile duct ligation in rats. Eur Surg Res. 2011;47(4):240-7. doi: 10.1159/000333087

30. Terzic D, Brmbolic B, Singer D, Dupanovic B, Korac $\mathrm{M}$, Selemovic D, et al. Liver enlargement associated with opportunistic infections in patients with human immunodeficiency virus infection. J Gastrointestin Liver Dis. 2008;17(4):401-4. url: http://www.jgld.ro/2008/4/6.pdf

31. Iwakiri Y. Pathophysiology of portal hypertension. Clin Liver Dis. 2014;18(2):281-91. doi: 10.1016/j.cld.2013.12.001 
32. Milesi-Halle A, Hendrickson HP, Laurenzana EM, Gentry WB, Owens SM. Sex- and dose-dependency in the pharmacokinetics and pharmacodynamics of $(+)$-methamphetamine and its metabolite (+)amphetamine in rats. Toxicol Appl Pharmacol. 2005;209(3):203-13. doi: 10.1016/j.taap.2005.04.007

33. Hambuchen MD, Ruedi-Bettschen D, Gunnell MG, Hendrickson H, Owens SM. Chronic treatment of $(+)$-methamphetamine-induced locomotor effects in rats using one or a combination of two high affinity anti-methamphetamine monoclonal antibodies. Hum Vaccin Immunother. 2016;12(9):2240-8. doi: 10.1080/21645515.2016.1179407

34. Schiorring E. Amphetamine induced selective stimulation of certain behaviour items with concurrent inhibition of others in an open-field test with rats. Behaviour. 1971;39(1):1-17. doi: 10.1163/156853971X00131

35. Mendelson J, Uemura N, Harris D, Nath RP, Fernandez E, Jacob P, et al. Human pharmacology of the methamphetamine stereoisomers. Clin Pharmacol Ther. 2006;80(4):403-20. doi: https://doi.org/10.1016/j.clpt.2006.06.013

36. Moszczynska A, Callan SP. Molecular, behavioral, and physiological consequences of methamphetamine neurotoxicity: implications for treatment. J Pharmacol Exp Ther. 2017;362(3):47488. doi: 10.1124/jpet.116.238501

37. Kitaichi K, Morishita Y, Doi Y, Ueyama J, Matsushima M, Zhao YL, et al. Increased plasma concentration and brain penetration of methamphetamine in behaviorally sensitized rats. Eur J Pharmacol. 2003;464(1):39-48. doi: 10.1016/S0014-2999(03)01321-9

38. Miller ML, Moreno AY, Aarde SM, Creehan KM, Vandewater SA, Vaillancourt BD, et al. A methamphetamine vaccine attenuates methamphetamine-induced disruptions in thermoregulation and activity in rats. Biol Psychiatry. 2013;73(8):721-8. doi: 10.1016/j.biopsych.2012.09.010

39. Hambuchen MD, Ruedi-Bettschen D, Williams DK, Hendrickson H, Owens SM. Treatment of rats with an anti-(+)-methamphetamine monoclonal antibody shortens the duration of action of repeated (+)methamphetamine challenges over a one month period. Vaccine. 2014;32(47):6213-9. doi: 10.1016/j.vaccine.2014.09.025

40. Quinn M, McMillin M, Galindo C, Frampton G, Pae HY, DeMorrow S. Bile acids permeabilize the blood brain barrier after bile duct ligation in rats via Rac1dependent mechanisms. Dig Liver Dis. 2014;46(6):527-34. doi: 10.1016/j.dld.2014.01.159

41. Schindler CW, Bross JG, Thorndike EB. Gender differences in the behavioral effects of methamphetamine. Eur J Pharmacol. 2002;442(3):231-5. doi: $10.1016 / \mathrm{S} 0014-$ 2999(02)01550-9

42. Milesi-Halle A, McMillan DE, Laurenzana EM, Byrnes-Blake KA, Owens SM. Sex differences in $(+)$-amphetamine- and (+)-methamphetamineinduced behavioral response in male and female Sprague-Dawley rats. Pharmacol Biochem Behav. 2007;86(1):140-9. doi: 10.1016/j.pbb.2006.12.018

43. Milesi-Halle A, Hambuchen MD, McMillan DE, Michael Owens S. The pharmacokinetics of methamphetamine self-administration in male and female rats. Drug Alcohol Depend. 2015;150:164-9. doi: 10.1016/j.drugalcdep.2015.02.032

44. Kogachi S, Chang L, Alicata D, Cunningham E, Ernst $T$. Sex differences in impulsivity and brain morphometry in methamphetamine users. Brain Struct Funct. 2017;222(1):215-27. doi: 10.1007/s00429-016-1212-2. 